\title{
Fontes de exposição humana ao chumbo no Brasil
}

\section{Lead sources of human exposure in Brazil}

\author{
Eduardo M. De Capitani', Mônica M. B. Paoliello², Glauce R. Costa de Almeida ${ }^{3}$
}

\begin{abstract}
RESUMO
Como resultado do crescimento da contaminação ambiental, houve um aumento do interesse relacionado ao acúmulo de metais pesados no corpo humano e seus efeitos danosos. Alguns desses metais, tal como o como chumbo, são elementos não essenciais e exercem efeitos tóxicos no sistema biológico. Apesar das medidas de controle estabelecidas por lei, intoxicações agudas e crônicas por chumbo ainda acontecem no Brasil. Algumas fontes de exposição a este metal são bem conhecidas, como por exemplo, atividades industriais como mineração, além de fábricas de reciclagem de baterias. No entanto, outras fontes, apesar de comuns, são completamente desconhecidas da população. Este artigo visa apresentar uma revisão da literatura passada e atual sobre as diversas fontes de exposição humana ao chumbo no Brasil.
\end{abstract}

Palavras-chave: Chumbo. Fontes de Exposição. Exposição Ambiental. Brasil.

$\mathrm{A}_{\mathrm{p}}^{\mathrm{s}}$ fontes de exposição humana ao chumbo podem ser divididas, grosso modo, em ocupacionais e não ocupacionais. Tal divisão tem cunho eminentemente prático, refletindo diferenças quanto à avaliação qualitativa e quantitativa da exposição, a magnitude, e as propostas de controle. Em várias situações a fonte de contaminação ocupacional extravasa os limites fabris e passa a ter importância na avaliação de exposição tida como não ocupacional. Como exemplos, temos as situações de contaminação peri-fabris, e ao redor de minerações, envolvendo moradores dessas áreas. A contaminação do ambiente domiciliar por chumbo, por meio de material particulado trazido pelos adultos da casa empregados em setores industriais que manipulam chumbo, é bastante conhecida e estudada e promove contaminações de magnitude elevada nas crianças da casa, principalmente de idade abaixo de seis anos. ${ }^{1}$

Inúmeras são as fontes potenciais de exposição humana a chumbo, variando de fontes industriais reconhecidas, até exposições inadvertidas, por meio de o consumo alimentar e de bebidas alcoólicas, uso de cosméticos, brinquedos, medicamentos herbáceos, e de terapêuticas tradicionais chinesas e indianas. $\mathrm{O}$ Quadro 1 lista as fontes mais importantes de chumbo, com as quais poderemos estar em contato de forma mais ou menos intensa dependendo das circunstâncias de trabalho, moradia e consumo.
1. Docente. Departamento de Clínica Médica. Faculdade de Ciências Médicas - Unicamp.

2. Docente. Departamento de Patologia Aplicada, Análise Clínicas e Toxicológicas. Universidade Estadual de Londrina - UEL.

3. Pós-Doutoranda. Departamento de Morfologia, Estomatologia e Fisiologia. Faculdade de Odontologia de Ribeirão Preto - USP.
Correspondência:

Glauce R. Costa de Almeida Departamento de Morfologia, Estomatologia e Fisiologia Faculdade de Odontologia de Ribeirão Preto, FORP/USP Av. do Café, S/N, Monte Alegre 14040-904, Ribeirão Preto-SP, Brasil. Telefone: (16) 36024065 / Fax: (16) 3602-4102; e-mail: glauce79@yahoo.com.br

Artigo recebido em 05/07/2009 Aprovado em 08/12/2009 
Quadro 1. Principais fontes ocupacionais e não ocupacionais de contaminação individual por compostos à base de chumbo

\section{Fontes Industriais}

Fundições Primárias (refino de minério de chumbo) e Secundárias (fusão de sucatas ou barras de chumbo para fins variados)

Produção de ligas (bronze, latão)

Fabricação e recuperação de baterias

Esmaltação de cerâmicas

Fabricação de pigmentos e "fritas" para cerâmicas

Fabricação de PVC e outros plásticos

Indústria de borracha

Fabricação de cabos elétricos (elemento dielétrico)

Operação de corte e solda de peças e chapas metálicas contendo chumbo

Jateamento de areia de estruturas metálicas pintadas com tintas com chumbo (pontes, navios)

Solda eletrônica $(\mathrm{Sn}+\mathrm{Pb})$

Produção de compostos orgânicos de chumbo

\section{Fontes não industriais ou não ocupacionais}

Bebidas alcoólicas (vinhos e destilados)

Uso de cristais finos e porcelana esmaltada

Utensílios de PVC

Fabricação caseira de "chumbadas" de pesca e cartuchos

Tinturas de cabelo

Prática de tiro ao alvo

Cerâmica artística caseira

Projétil de arma de fogo alojado em articulações ou canal medular

Alimentos industrializados

Tintas em brinquedos

Medicina chinesa e indiana

O chumbo é um metal com largo uso industrial em função de suas características físico químicas, como baixo ponto de fusão $\left(327^{\circ} \mathrm{C}\right)$, densidade elevada, maleabilidade, altamente dielétrico, resistência a radiações ionizantes e formação de ligas com outros metais. Todas as ligas metálicas formadas com estanho, cobre, arsênio, antimônio, bismuto, cádmio e sódio apresentam importantes aplicações industriais, como por exemplo, soldas, fusíveis, material de tipografia, material de antifricção e revestimentos de cabos elétricos. A ductilidade única do chumbo o torna particularmente apropriado para sua utilização como forro para cabos de telefone e de televisão, pois ele pode ser estirado para formar um revestimento contínuo em torno dos condutores internos.

Desde a antiguidade o chumbo é fundido e utilizado sozinho ou junto a outros metais na fabricação de armas e utensílios domésticos como copos, taças e recipientes para líquidos e alimentos, além de aquedutos e reservatórios para fermentação e acondicionamento de bebidas alcoólicas. Durante o império romano foi utilizado, na forma de óxidos, na conservação e correção do gosto de vinhos, sendo este um hábito mantido por pequenas vinícolas européias até poucas décadas atrás. Além disso, os romanos utilizavam o metal para a fabricação de canos, no século III a.C. e, ainda hoje, esses canos de chumbo (Figura 1) continuam presentes em casas mais antigas e outras edificações erguidas antes de 1978. Os antigos egípcios utilizaram o chumbo há mais de oito mil anos. Os jardins suspensos da Babilônia eram assoalhados com folhas de chumbo soldadas, e as pedras das pontes eram ligadas por ganchos de ferro soldados com chumbo.

A Figura 2 mostra algumas aplicações do chumbo fundido, como por exemplo, a "chumbada" para pesca (Figura 2A) e o peso para balanceamento de rodas (Figura 2B).

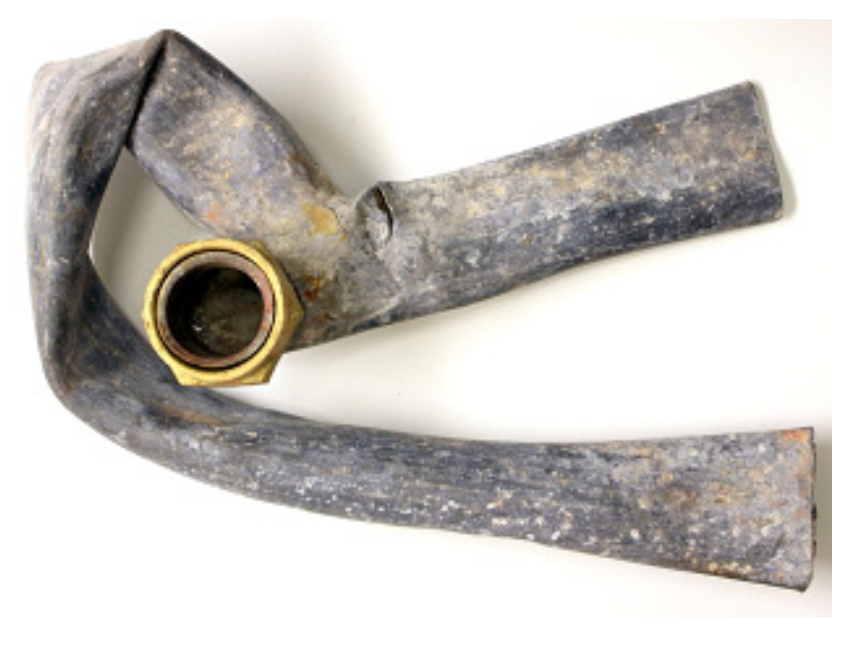

Figura 1. Canos de chumbo utilizados em casas antigas. 

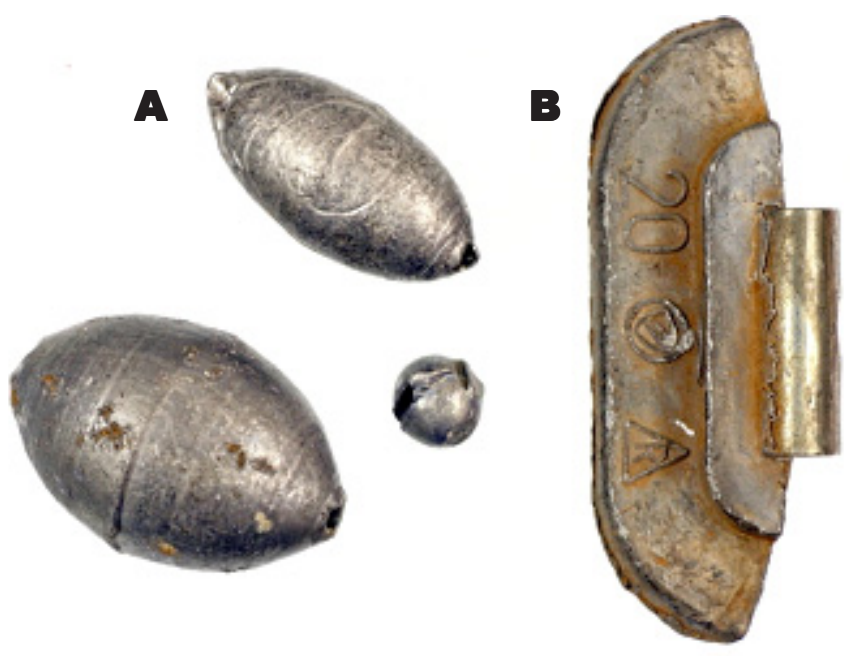

Figura 2. Aplicações do chumbo fundido: $2 A$ - "chumbada" utilizada em pescarias; 2B: contrapeso para balanceamento de rodas.

No Brasil, a produção de chumbo metálico foi importante até a década de 90, quando duas grandes fundições primárias utilizadas no refino do minério extraído na Bahia e em Adrianópolis, PR fecharam suas atividades industriais em 1996. Desde então a produção nacional do metal tem declinado, representando não mais de $8 \%$ do total de chumbo consumido no país por ano. ${ }^{2}$ Atualmente calcula-se que cerca de $90 \%$ do consumo nacional de chumbo metálico provenha de atividades de reciclagem. ${ }^{3}$ Os maiores consumidores de chumbo no país são as indústrias de baterias automotivas (80\%), seguidos das indústrias de pigmentos com $12 \%$ e o setor eletro-eletrônico (solda e ligas) com $8 \% .^{4}$

Thorton et al. $(1995)^{5}$ estimaram que cerca de um terço da exposição diária de uma população urbana ao chumbo venha de fontes atmosféricas, pois fontes móveis e estacionárias de particulado contendo chumbo tendem a se estabelecer em centros urbanos densamente populosos. Ou próximo a fundições e refinarias de metais não ferrosos. ${ }^{6}$

Um estudo realizado na cidade do Rio de Janeiro em área próxima a uma recicladora de baterias automotivas mostrou concentrações de chumbo no ar atmosférico variando de 0,07 a $183,3 \mu \mathrm{g} / \mathrm{m}^{3}$ em amostras distando 25 a 500 metros da fonte de emissão. ${ }^{7}$ Considerando o valor limite de $1,5 \mu \mathrm{g} / \mathrm{m}^{3}$ estabelecido pela agência de proteção ambiental norte americana (USEPA) para chumbo no ar constatou-se que em $50 \%$ das amostras coletadas a 25 metros da indústria esse limite havia sido ultrapassado. Nesse mesmo es- tudo, os autores avaliaram as concentrações de chumbo na poeira doméstica e peri-domicilar e constataram que $50 \%$ das amostras coletadas no interior das casas excediam o limite de $1500 \mu \mathrm{g} / \mathrm{m}^{2}$, em habitações no perímetro de $50 \mathrm{~m}$ da fonte. ${ }^{7}$

Como parte da investigação de vias de exposição a chumbo de crianças moradoras próximas a fundições primária e secundária de chumbo, respectivamente em Adrianópolis, Paraná e Bauru, SP, De Capitani et al. ${ }^{8}$ (dados não publicados), constataram concentrações elevadas de chumbo em poeira domiciliar de casas dentro de um perímetro de até 800 metros das fontes de contaminação (notar que à época da amostragem, ambas a empresas estavam inativadas). Nas proximidades da fundição primária de chumbo os níveis na poeira variaram de 299 a 3.268 $\mu \mathrm{g} / \mathrm{g}(\mathrm{ppm})$. Ao redor da fundição secundária de chumbo em Bauru, SP, as concentrações variaram de 702 a $4.597 \mu \mathrm{g} / \mathrm{g}(\mathrm{ppm}) .{ }^{8}$ Apesar de não haver consenso sobre o limite máximo de chumbo em poeira doméstica relacionado com prevenção de efeitos adversos, a agência USEPA preconiza como valor limite guia para solo a concentração de $400 \mu \mathrm{g} / \mathrm{g}$ (ppm) ${ }^{6}{ }^{6}$ e vários estudos têm associado concentrações elevadas de chumbo em poeira doméstica com níveis elevados de chumbo em sangue de crianças. ${ }^{9,10,11}$

O chumbo é um excelente metal quando usado para proteger da corrosão atmosférica devido a sua rápida oxidação superficial em forma de película de óxido, formando o protóxido de chumbo. Graças a essa resistência a corrosão, este metal encontra muitas aplicações na indústria de construção e, principalmente, na indústria química. A folha de chumbo, um dos mais antigos e mais duráveis materiais de cobertura, é reconhecida por durar, no mínimo, duzentos anos. Esta folha de chumbo fundido é produzida por firmas especializadas e é largamente usada para a substituição de folha de chumbo antiga em catedrais e igrejas onde a autenticidade é importante. Nos edifícios, a folha de chumbo é, geralmente, fixada exteriormente e assim fica sujeita a condições de variação de temperatura. Devido o elevado coeficiente de expansão térmica linear do metal, quando a diferença entre a temperatura do verão e do inverno é levada em consideração, o resultado de um cálculo simples demonstra o aumento das dimensões da folha.

O chumbo tem sido usado largamente até anos recentes em pigmentos utilizados em cerâmicas e porcelanas utilitárias e decorativas. Poucos estudos no Brasil avaliaram tal uso como fonte potencial de con- 
taminação das pessoas por meio do uso dessas cerâmicas e porcelanas na alimentação diária, por exemplo. Em estados do nordeste o uso de cerâmicas vitrificadas com chumbo é bastante difuso e constitui prática comum, como por exemplo em Caruaru, PE. O óxido de chumbo é o composto mais utilizado. Lima et al. (2002) mediram a concentração de chumbo em peças vitrificadas antes e depois do processo de queima e constataram valores de 211 a $13.660 \mathrm{mg} / \mathrm{L}$ de chumbo sendo liberado nesse processo, estimando uma perda média de $166 \mathrm{~kg}$ de chumbo por ano no meio ambiente. ${ }^{12}$ A poluição do ar atmosférico, principalmente urbano durante grande parte do século 20 deveu-se à adição na gasolina do composto orgânico chumbo-teraetila como antidetonante e para elevar a octanagem. Descontando-se as fontes industriais, estima-se que $90 \%$ das emissões de chumbo na atmosfera no mundo ainda são devidas ao chumbo na gasolina. Atualmente o chumbo utilizado na gasolina representa $2,2 \%$ do consumo total de chumbo no mundo, representando uma tendência global de redução a partir das décadas de 70 e 80 em vários países. ${ }^{13}$ Até então, quase toda a gasolina consumida no mundo continha chumbo em concentrações ao redor de $0,4 \mathrm{~g} / \mathrm{L} .{ }^{13}$

No Brasil a diminuição das concentrações de chumbo na gasolina começou como consequência do programa de produção de álcool anidro em 1975, que visava estrategicamente uma menor dependência da importação de petróleo. A partir da implementação do programa, alterações nos motores dos carros foram efetivadas visando à substituição do chumbo-tetraetila (composto importado e caro) por percentual de etanol. Em 1979 a Resolução 14/79 do Conselho Nacional do Petróleo (CNP) definiu a gasolina de tipo C (usada em automóveis e embarcações) como isenta de chumbo-tetraetila e contendo $22 \%$ em volume de etanol anidro. As gasolinas de tipo A e B, de alta octanagem, utilizadas pelas Forças Armadas continuaram a ser produzidas com a adição de chumbo-tetraetila. Em 1982 assistiu-se a um retrocesso nessa regulamentação, voltando-se legalmente ao status anterior a 1979, porém, a partir de 1993, através da lei 7823/93 estabelece-se a obrigatoriedade de adição de $22 \%$ de etanol anidro à gasolina, tornando desnecessário, na prática, a adição de chumbo-tetraetila. Apesar dessas indefinições legais, Romano et al. (1992) avaliando concentrações de chumbo no ar atmosférico no período de 1978 a 1987 na cidade de São Paulo, mostra que houve marcante declínio nesse período (Figura 3). ${ }^{14}$ 


\section{Tabela 1}

Concentrações de chumbo [mg/kg] em solos em áreas contaminadas próximas a fundições primárias e secundárias no Brasil, e valores de intervenção estabelecidos para áreas agrícolas, urbanas residenciais e industriais

$[\mathrm{Pb}]$ no solo encontrados em áreas residenciais

Valores de intervenção estabelecidos segundo o uso do solo

Agricultura Residencial Parques urbanos Industrial

Bahia (Santo Amaro de Purificação) até 7.500

São Paulo (Bauru)

$50-1000^{*}$

Paraná (Adrianópolis)

$117-940 * *$

Brasil (CETESB São Paulo)

375

350

1.200

Canadá

375

500

1.000

Alemanha

$400^{\#}$

$200^{\#}$

$2000^{\#}$

* solo de 0 a $2 \mathrm{~cm}$ de profundidade

** solo de 0 a $5 \mathrm{~cm}$ de profundidade

\# valores de intervenção considerando ingestão direta do solo por crianças

tante baixos de contaminação de águas superficiais, e praticamente ausência de consumo de água potável contaminada, na maioria das áreas até o momento estudadas. ${ }^{13,15,18,19}$

Sessenta e nove amostras de águas minerais comercializadas no país foram estudadas por Maio et al. (2002). O chumbo, e outros metais de interesse toxicológico, não foram detectados. ${ }^{20}$

Os alimentos e as bebidas sempre foram motivo de preocupação quanto à possível contaminação por chumbo ao longo da história. Produtos agrícolas produzidos em solos contaminados ou mesmo irrigados com águas contaminadas tendem a incorporar chumbo em maior ou menor proporção dependendo de variáveis como $\mathrm{pH}$ do solo e da água, forma química do chumbo (óxidos mais solúveis, sulfetos menos solúveis, por exemplo), espécie vegetal (cada espécie tem potencial de fixação de chumbo diferente). Outra preocupação refere-se aos alimentos industrializados. Praticamente todo alimento que passa por processo de industrialização entra em contato direto com reservatórios, reatores, encanamentos, etc., feitos com ligas metálicas não necessariamente isentas de chumbo (ligas como o bronze e o latão, por exemplo, contém ao redor de 4 a $5 \%$ de chumbo em sua composição), sem contar com soldas e peças de vedação e encaixe. A única liga metálica com certeza isenta de chumbo é o aço inoxidável, daí a exigência recente de uso desse metal nos reservatórios e circuitos de produção (destiladores, alambiques, etc.) e distribuição de bebidas alcoólicas e alimentos processados e restrição de uso de soldas a base de chumbo. Faz parte da história a contaminação que ocorria em alimentos enlatados em função da solda a base de chumbo que vedava as mesmas. Atualmente o processo prescinde de solda com chumbo ou outros metais, pois a vedação é feita em processo de fusão a quente das partes metálicas e a superfície das latas é protegida por filme plástico que evita o contato dos alimentos ou sucos com o metal. A avaliação das concentrações de chumbo em alimentos nos EUA, pela USEPA, mostram que os produtos com maiores teores desse metal são os vegetais, as frutas e os sucos de frutas seguidos por carnes, peixes e frangos e grãos e cereais (Tabela 2). ${ }^{21}$ Esses dados, longe de serem preocupantes com relação ao risco de intoxicação clínica, demonstram o quão difusa é a contaminação por chumbo em todo o meio ambiente, dependendo, é claro, de condições regionais e nacionais específicas.

No Brasil, em 1998, o Ministério da Saúde publicou portaria regulando concentrações máximas de chumbo em alimentos, utilizando dados internacionais sobre limites já estabelecidos nos EUA e na Comunidade Européia (Tabela 3). Resultados de estudos sobre contaminação de alimentos por chumbo no Brasil têm sido publicados com certa frequência a partir de instituições responsáveis pela vigilância sanitária e epidemiológica. Em 1997, Okada et al (1997) dosaram chumbo em amostras de leite in natura e pasteurizado produzido em área próxima à fundição secundária de chumbo no Vale do Rio Paraíba do Sul em São Paulo, mostrando que $20 \%$ das amostras encontravam-se acima do limite permitido para consumo. ${ }^{22}$ 


\section{Tabela 2}

Concentrações de chumbo em alimentos nos EUA derivadas de dados da agência de proteção ambiental (US-EPA)

\begin{tabular}{lc}
\hline Alimentos & {$[\mathrm{Pb}] \mathrm{em} \mathrm{ng} / \mathrm{g}$} \\
\hline Laticínios & $3-83$ \\
Carnes, peixes, frangos & $2-159$ \\
Grãos e cereais & $2-136$ \\
Verduras e legumes & $5-649$ \\
Frutas e sucos de frutas & $5-223$ \\
Óleos e gorduras & $8-28$ \\
Açúcar & $6-73$ \\
Bebidas não alcoólicas $(\mu \mathrm{g} / \mathrm{L})$ & $2-41$ \\
\hline
\end{tabular}

\section{Tabela 3}

Limites máximos permitidos para alimentos por portaria do Ministério da Saúde (MS-685/1998)

\begin{tabular}{lc}
\hline Tipo de alimento & $\begin{array}{c}\text { Limite máximo de chumbo } \\
\mathrm{mg} / \mathrm{kg}[\mathrm{ppm}]\end{array}$ \\
\hline Óleos, gorduras, banhas & 0,1 \\
Balas e caramelos & 2,0 \\
Grãos de cacau & 2,0 \\
Chocolates adocicados & 1,0 \\
Dextrose (glicose) & 2,0 \\
Sucos de cítricos & 0,3 \\
Leite pasteurizado & 0,05 \\
Peixes e produtos derivados & 2,0 \\
Alimentos para crianças & \\
abaixo de 3 anos & 0,2
\end{tabular}

Com relação a frutos do mar, em área de influência da bacia do rio Ribeira de Iguape, que aparentemente sofre as consequências da mineração e refino de chumbo em sua parte alta (Adrianópolis, PR), ostras de criadouros no município de Cananéia, já na foz desse rio, foram analisadas e mostram-se dentro dos limites estabelecidos (média de chumbo de $0,08 \mathrm{mg} /$ $\mathrm{kg}$, com valor máximo de $0,17 \mathrm{mg} / \mathrm{kg}$ ). ${ }^{23}$ No estuário de Santos e São Vicente, São Paulo, região mais impactada pela poluição industrial, mas sem fontes importantes de contaminação por chumbo como fundições e refinarias de minério, análise de chumbo em bivalves incluindo ostras, também não mostrou níveis acima dos recomendados. ${ }^{24}$
Verduras e legumes comercializados no estado de São Paulo foram analisados quanto às concentrações de chumbo e demonstrou-se não ser risco de contaminação importante para os consumidores. ${ }^{25}$

Os plásticos e, principalmente, o $\mathrm{PVC}$, onde o chumbo age como estabilizante na polimerização e pode também estar presente em pigmentos coloridos, podem ser fontes de contaminação de alimentos conservados em recipientes feitos com esses materiais. Em análise de 997 amostras de pigmentos de plásticos usados em acondicionadores de alimentos no mercado brasileiro no período de 1982-1989, Garrido et al (1991) encontraram 5,4\% das amostras com concentrações de chumbo acima de $0,01 \%$ como preconiza a legislação. ${ }^{26}$ Em produtos escolares, os mesmos autores encontraram chumbo acima dos limites permitidos em diversos produtos que são costumeiramente colocados na boca pelas crianças, como lápis (grafite), borrachas e giz de cera (Tabela 4$).{ }^{27}$

\section{Tabela 4}

Concentração de chumbo em artigos escolares amostrados aleatoriamente no comércio

\begin{tabular}{lc}
\hline Artigo & $\begin{array}{c}\text { Concentração de chumbo } \\
\text { em mg/kg (ppm) }\end{array}$ \\
\hline Borrachas & $0,06-249$ \\
Giz de cera & $0,10-5.000$ \\
Tinta & $0,07-46$ \\
Crayons & $1,88-14$ \\
Massa de modelagem & $0,5-12$ \\
Cola colorida & $0,09-1,4$ \\
\hline
\end{tabular}

* Limite máximo permitido para tintas de brinquedos $=100 \mathrm{mg} / \mathrm{kg}$ Resolução MS-105/99. Fonte: (Garrido et al., 1991)

O chumbo também pode estar presente em alguns corantes. Os pigmentos de amarelos de cromo são constituídos de cromato de chumbo e/ou de uma solução sólida de cromato de chumbo e sulfato de chumbo, em proporções diferentes, de acordo com a coloração que se deseja. No século 19, esses pigmentos de amarelos de cromo eram utilizados pelos padeiros e confeiteiros como um agente corante. Em 1887, David Stewart relatou em "Medical News" a intoxicação de nove membros de uma mesma família pelo cromato de chumbo utilizado como corante de pães. ${ }^{28}$ Essa substância também foi causa da morte duas crianças, em 1889, as quais ingeriram doces que continham amarelo de cromo. ${ }^{29}$ 
Apesar da legislação sobre chumbo em tintas de brinquedos, grande preocupação é com a falta de controle de importação de brinquedos asiáticos, principalmente chineses que não são adequados aos limites estabelecidos.

O chumbo também está presente no tabaco, em concentrações de aproximadamente 2,5 - 14,2 $\mu \mathrm{g}$ por cigarro, dos quais cerca de $2-6 \%$ podem ser efetivamente inalados pelo fumante (inclusive o fumante passivo). ${ }^{30} \mathrm{~A}$ fumaça do cigarro é uma mistura de cerca de 4.700 substâncias tóxicas diferentes, apresentando uma fase gasosa e outra particulada. A fase gasosa é composta, entre outros, por monóxido de carbono, amônia, cetonas, formaldeído, acetaldeído e acroleína. A fase particulada contém nicotina e alcatrão, que concentra 43 substâncias cancerígenas, dentre as quais estão o arsênio, níquel, benzo-pireno, cádmio e chumbo. ${ }^{31}$

Durante muito tempo se tem empregado o chumbo como manta protetora para os aparelhos de raios$\mathrm{X}$, devido sua propriedade singular de absorver radiações de ondas curtas. Em virtude das aplicações cada vez mais intensas da energia atômica, torna-se cada vez mais importante as aplicações do chumbo como blindagem contra a radiação. Além disso, no filme radiográfico intra-oral, entre seus envoltórios, existe uma fina folha de chumbo com um padrão em escamas, que possui a função de reduzir a radiação secundária e a exposição do paciente. No entanto, muitos odontopediatras, desconhecendo os riscos do chumbo, entregam essas folhas para seus pacientes brincarem.

Remédios alternativos procedentes de culturas médicas e terapêuticas exóticas, como medicina tradicional chinesa e a medicina indiana Ayurveda, po- dem conter concentrações de chumbo e outros metais, capazes de produzir quadros clínicos de intoxicação ou acúmulo de metais cronicamente. A literatura internacional tem mostrado casos dramáticos de intoxicação a partir do uso desses terapêuticos, alertando para a necessidade de cuidado adicional por parte do consumidor. ${ }^{32-35}$ No Brasil não dispomos de dados sobre esse tipo de contaminação e nenhum estudo foi realizado nesse sentido até o momento. Outras práticas terapêuticas como o uso de greta ou azarcon, na medicina tradicional mexicana no tratamento de problemas gastrointestinais de crianças, ou o uso de paylooah proveniente do sudeste asiático, para tratamento de febres e rash cutâneo, podem conter concentrações de chumbo elevadas. ${ }^{36}$ Bali goli, coral, ghazard, liga e rueda, são nomes de remédios tradicionais exóticos que podem conter chumbo. ${ }^{36}$ Proveniente do Oriente Médio, um cosmético utilizado nos olhos como delineador, chamado Kohl ou suma, originalmente feito com antimônio, contém chumbo, e seu uso está associado à elevação nos níveis de chumbo no sangue de usuárias frequentes. ${ }^{37,38}$

O acréscimo de substância estranha a drogas ilícitas por traficantes é bastante comum e tem sido relatada com frequência, principalmente no comércio ilegal de cocaína e heroína. Recente epidemia de intoxicações clínicas por chumbo, com níveis de plumbemia elevados e sinais e sintomas compatíveis, foi observada em população de estudantes universitários na Alemanha que haviam consumido maconha comercializada por vendedor que acrescentava chumbo metálico fragmentado ao produto visando aumentar seu peso, colocando o consumo de maconha como potencial fonte de contaminação por chumbo. ${ }^{39}$

\begin{abstract}
As result of increased environmental contamination, the interest on the accumulation of heavy metals in the human body and its harmful effects has increased. Some metals, such as lead, are non-essential elements and exert toxic effects on biological system. Despite the control measures established by law, acute and chronic intoxication by lead still happen in Brazil. Some sources of lead exposure are well known, such as industrial activities like mining and factories for batteries recycling. However, other sources, although common, are completely unknown by population. This article aims to present a review of past and current literature on the various lead sources of human exposure in Brazil.
\end{abstract}

Keywords: Lead. Sources of Exposure. Environmental Exposure. Brazil. 


\section{Referências Bibliográficas}

1. Grandjean P, Bach E. Indirect exposures: the significance of bystanders at work and at home. Am Ind Hyg Assoc J. 1986; 47:819-24.

2. Paoliello MM, De Capitani EM. Occupational and environmental human lead exposure in Brazil. Environ Res. 2007; 103: 288-97.

3. Franco-Netto G, Alonzo HG, Cancio J, Jost M, de SouzaOliveira S. Human health risk reduction due to lead exposure in Brazil. Salud Pública Mex. 2003; 45 Suppl 2:S255-8.

4. Brasil. Balanço mineral brasileiro. Chumbo. In: DNPM, editor: DNPM-MME, 2001.

5. Thornton I, Culbard EB, Moorcroft S, Watt JM, Wheatley M, Thompson M, et al. Metals in urban dusts and soils. Environ Technol. 1985; 6:137-44.

6. ATSDR. Toxicologic Profile for Lead - Update Statement. In: Registry USDoHHS-PHS-AfTSaD, editor. Atlanta, 1999: 587.

7. Quitério SL, Silva CRS, Vaitsman DS, Martinhon PT, Moreira MFR, Araujo UC, et al. Use of dust and air as indicators of environmental pollution in areas adjacent to a source of stationary lead emission. Cad Saúde Publica. 2001;17:501-8.

8. De Capitani EM, Ensweiler JMSA, Paoliello MMB. Dados não publicados, 2009.

9. Meyer I, Heinrich J, Trepka MJ, Krause C, Schulz C, Meyer E, et al. The effect of lead in tap water on blood lead in children in a smelter town. Sci Total Environ. 1998; 209:255-71.

10. Trepka MJ, Heinrich J, Krause C, Schulz C, Lippold U, Meyer $E$, et al. The internal burden of lead among children in a smelter town—a small area analysis. Environ Res. 1997; 72: 118-30.

11. Lanphear BP, Matte TD, Rogers J, Clickner RP, Dietz B, Bornschein RL, et al. The contribution of lead-contaminated house dust and residential soil to children's blood lead levels. A pooled analysis of 12 epidemiologic studies. Environ Res. 1998; 79:51-68.

12. Lima M, Pimentel M, Brayner F, Abreu C. Lead released by handmade glazed ceramic food wares. Rev Bras Toxicol. 2002;15:19-23

13. Paoliello MM, De Capitani EM. Environmental contamination and human exposure to lead in Brazil. Rev Environ Contam Toxicol. 2005; 184:59-96.

14. Romano J, Godinho R, Alonso C, Martins M. Ethanol induced changes in the atmospheric lead in São Paulo Metropolitan Area, Brazil. 9th Word Air Clean Congress 1992.

15. Freitas CU. Vigilância de população exposta a chumbo no município de Bauru, São Paulo: investigação de fatores de risco de exposição e avaliação da dinâmica institucional. Universidade de São Paulo, 2004.

16. Paoliello MM, De Capitani EM, da Cunha FG, Matsuo T, Carvalho Mde F, Sakuma A, et al. Exposure of children to lead and cadmium from a mining area of Brazil. Environ Res. 2002; 88: 120-8.

17. Silvany-Neto AM, Carvalho FM, Tavares TM, Guimaraes GC, Amorim CJ, Peres MF, et al. Lead poisoning among children of Santo Amaro, Bahia, Brazil in 1980, 1985, and 1992. Bull Pan Am Health Organ. 1996;30:51-62.

18. Jordão CP. Distribution of heavy metals in environmental samples near smelters and mining areas in Brazil. Environ Technol. 1999; 20:489-98.

19. Cunha FG. Contaminação humana e ambiental por chumbo no Vale do Ribeira, nos Estados de São Paulo e Paraná, Brasil. Universidade Estadual de Campinas, 2003.

20. Maio F, Okada I, Carvalho M, Kira C, Duran M, Zenebon O. Evaluation of the labelling, the minerals and inorganic con- taminants in domestic and imported mineral water. Rev Inst Adolfo Lutz. 2002; 61:227-32.

21. USEPA. Assessing human health risks from chemically contaminated fish and shellfish: a guidance manual. Washington DC, 1989.

22. Okada IA, Sakuma AM, Maio FD, Dovidauskas S, Zenebon O. [Evaluation of lead and cadmium levels in milk in environmental contamination in the Vale do Paraiba Region, southeastern Brazil]. Rev Saude Publica. 1997; 31:140-3.

23. Machado I, Maio F, Kira C, Carvalho M. Pb, Cd, Hg, Cu and Zn in mangrove oyster Crassostrea brasiliana Cananéia estuary, São Paulo, Brazil. Rev Inst Adolfo Lutz. 2002; 61:13-18.

24. Pereira O, Henriques M, Zenebon O, Sakuma A, Kira C. Determination of $\mathrm{Pb}, \mathrm{Cd}, \mathrm{Hg}, \mathrm{Cu}$ and $\mathrm{Zn}$ levels in molluscus (Crassostrea brasiliana, Perna perna and Mytella falcate) Rev Inst Adolfo Lutz. 2002; 61:19-25.

25. Sakuma A, Scorsafava M, Zenebon O, Tiglea P, Fukumoto C. Hortaliças comercializadas em São Paulo: aspectos da contaminação por chumbo, cádmio e zinco. Rev Inst Adolfo Lutz. 1989; 49:81-4.

26. Garrido NS, Pregnolatto NP, Murata LTF, Silva MR, Nunes MCD, Antunes JLF, et al. Avaliação dos níveis de arsênio, chumbo e cádmio em corantes e pigmentos utilizados em embalagens para alimentos no período de 1982 a 1989. Rev Inst Adolfo Lutz. 1991; 51:63-8.

27. Garrido NS, Pregnolato NP, Murata LTF, Silva MR, Nunes MCD, Engler VM, et al. Determinação de chumbo e cádmio em artigos escolares. Rev Inst Adolfo Lutz. 1990; 50:291-6.

28. Stewart DD. Notes on some obscure cases on poisoning by lead chromate manifested chiefly by encephalopathy. Med News 1887; 1: 676-81.

29. Glenn W. Chrome yellow considered as a poison. Science 1889;13:347-49.

30. Ajab H, Yasmeen S, Yaqub A, Ajab Z, Junaid M, Siddique M, et al. Evaluation of trace metals in tobacco of local and imported cigarette brands used in Pakistan by spectrophotometer through microwave digestion. J Toxicol Sci. 2008; 33: 415-20.

31. Brasil, Ministério da Saúde. Secretaria Nacional de Assistência à Saúde. Instituto Nacional do Câncer. Coordenação Nacional de Controle de Tabagismo e Prevenção Primária de Câncer (Contapp). Falando sobre Tabagismo. Ministério da Saúde. Rio de Janeiro: 3oe ed. 1998.

32. Elevated blood lead levels among internationally adopted children - United States, 1998. MMWR Morb Mortal Wkly Rep 2000; 49:97-100.

33. Kaul B. Lead exposure and iron deficiency among Jammu and New Delhi children. Indian J Pediatr. 1999;66:27-35.

34. Gogte ST, Basu N, Sinclair S, Ghai OP, Bhide NK. Blood lead levels of children with pica and surma use. Indian J Pediatr. 1991; 58:513-9.

35. Dunbabin DW, Tallis GA, Popplewell PY, Lee RA. Lead poisoning from Indian herbal medicine (Ayurveda). Med J Aust 1992; 157:835-6.

36. Hayes EB. The hazard of lead in children. In: Brooks SM, editor. Environmental Medicine. St Louis: Mosby, 1995: 383-97.

37. Al-Ashban RM, Aslam M, Shah AH. Kohl (surma): a toxic traditional eye cosmetic study in Saudi Arabia. Public Health. 2004; 118: 292-8.

38. Al-Saleh I, Nester M, DeVol E, Shinwari N, Al-Shahria S. Determinants of blood lead levels in Saudi Arabian schoolgirls. Int J Occup Environ Health 1999; 5: 107-14.

39. Busse F, Omidi L, Timper K, Leichtle A, Windgassen M, Kluge $\mathrm{E}$, et al. Lead poisoning due to adulterated marijuana. $\mathrm{N}$ Engl J Med. 2008;358:1641-2. 